\title{
SOME TESTS ON CPT INVARIANCE
}

\author{
C. Q. GENG* \\ Department of Physics, National Tsing Hua University \\ Hsinchu, Taiwan, 30043 \\ geng@phys.nthu.edu.tw \\ LEI GENG \\ Department of Applied Physics \\ University of Science and Technology Beijing \\ Beijing, 100083
}

\begin{abstract}
We first briefly review tests on $C P T$ invariance based on the consequences of the CPT theorem and then present some possible $C P T$ tests due to exotic models in which some of the CPT conditions are lost, such as those without hermiticity.

Keywords: Parity, Charge conjugation, Time-reversal, CPT, Photon

PACS Nos.: 11.30.Er, 14.70.Bh, 14.40.Aq
\end{abstract}

\section{Introduction}

Symmetry principles dictate the basic laws of physics, control structure of matter and define the fundamental forces in nature. The best known definition of Symmetry can be referred to the book by Hermann Weyl 1 , which stated as follows:

"Symmetry ... is an idea which has guided man through the centuries to the understanding and the creation of order, beauty and perfection."

One can relates symmetries and conservation laws from Noether's famous theorem that every continuous symmetry of a Lagrangian implies a conserved quantity and vice versa, i.e.,

$$
\text { Symmetries } \Longleftrightarrow \text { Conservation Laws . }
$$

Invariances or symmetries of translation in time, translation in space and rotation correspond to conservations of energy, momentum and angular momentum, respectively. In addition to continuous symmetries, there are discrete symmetries. In particle physics, there are three very important discrete symmetries, called $P, T$

*Talk presented at the International Conference on Non-Perturbative Quantum Field Theory: Lattice and Beyond. 
and $C$, where

$$
\begin{aligned}
P: & \text { parity or space inversion } \\
& \vec{x} \longleftrightarrow-\vec{x}, \\
T: & \text { time reversal } \\
& t \longleftrightarrow-t, \\
C: & \text { particle }(p)-\text { antiparticle }(\bar{p}) \text { exchange } \\
& \text { or charge conjugation } \\
& e^{-} \longleftrightarrow e^{+} .
\end{aligned}
$$

In the following table, various physical quantities under $P, T$ and $C$ transformations are given:

$\begin{array}{cllcllllll}\text { coordinate } & \vec{x} & \longrightarrow & -\vec{x} & \vec{x} & \longrightarrow & \vec{x} & \vec{x} & \longrightarrow & \vec{x} \\ \text { time } & t & \longrightarrow & t & t & \longrightarrow & -t & t & \longrightarrow & -t \\ \text { momentum } & \vec{p} & \longrightarrow & -\vec{p} & \vec{p} & \longrightarrow & -\vec{p} & \vec{p} & \longrightarrow & \vec{p} \\ \text { energy } & \varepsilon & \longrightarrow & \varepsilon & \varepsilon & \longrightarrow & \varepsilon & \varepsilon & \longrightarrow & \varepsilon \\ \text { angular momentum } & \vec{J} & \longrightarrow & \vec{J} & \vec{J} & \longrightarrow & -\vec{J} & \vec{J} & \longrightarrow & \vec{J} \\ \text { spin } & \vec{s} & \longrightarrow & \vec{s} & \vec{s} & \longrightarrow & -\vec{s} & \vec{s} & \longrightarrow & \vec{s} \\ \text { charge } & Q & \longrightarrow & Q & Q & \longrightarrow & Q & Q & \longrightarrow & -Q \\ \text { electric field } & \vec{E} & \longrightarrow & -\vec{E} & \vec{E} & \longrightarrow & \vec{E} & \vec{E} & \longrightarrow & -\vec{E} \\ \text { magnetice field } & \vec{B} & \longrightarrow & \vec{B} & \vec{B} & \longrightarrow & -\vec{B} & \vec{B} & \longrightarrow & -\vec{B}\end{array}$

From the above table, we can study the symmetry properties of $C, P$ and $T$ as well as their combined operations for various physical observables. For examples, $T$-odd product correlations of $\vec{v}_{1} \cdot\left(\vec{v}_{2} \times \vec{v}_{3}\right)\left(\vec{v}_{i}=\vec{s}_{i}\right.$ or $\left.\vec{p}_{i}\right)$ and $\vec{s} \cdot \vec{E}$ and $\vec{E} \cdot \vec{B}$ are used to probe $T$ violation for $\mathrm{K}$ and $\mathrm{B}$ decays and electric dipole moments of particles, respectively.

For a long time, physics laws were thought to be $C, P$ and $T$ conserved. With these conservation laws, many experimental phenomena can be understood. For example, charge conservation law allows ${ }^{1} S_{0}$ positronium atom decaying to $2 \gamma$, but forbids $3 \gamma$ mode, since $C(n \gamma)=(-1)^{n}$. Indeed, electromagnetic and strong interactions respect all these symmetries at very high accuracy. However, in 1956 Lee and Yang ${ }^{2]}$ concluded that $P$ must be violated in weak interaction and in 195734 both $P$ and $C$ were found to be violated maximally. To understand weak interaction, $V-A$ theory was proposed by Marshak et al. 5 in 1957. In this theory, $P$ and $C$ are violated maximally but the combined $C P$ operation is still conserved. In 1964, CP violation (CPV) ${ }^{6}$ was observed at the level of $O\left(10^{-3}\right)$ in the neutral kaon system. Recently, large CP violating effects have also been found in the neutral B decays ${ }^{17}$. Furthermore, some evidences of $T$ violation (TV) ${ }^{8}$ was also shown in the $K^{0}$ system. It should be noted that no CPV or TV has been seen in any charged systems yet, such as those of $K^{ \pm}$and $B^{ \pm}$. Furthermore, the origin of the violations 
in $K^{0}$ and $B^{0}$ systems remains unclear. In the standard model, CPV or TV arises from a unique physical phase in the Cabibbo-Kobayashi-Maskawa (CKM) quark mixing matrix 9 . On the other hand, at present, there is no any experimental sign of CPT violation. The experimental results can be summarized in Table 1 .

Table 1. Symmetry invariance and violation

\begin{tabular}{|c|c|c|c|c|c|}
\hline Forces & $P$ & $C$ & $C P$ & $T$ & $C P T$ \\
\hline \hline Gravity & $\sqrt{ }$ & $\sqrt{ }$ & $\sqrt{ }$ & $\sqrt{ }$ & $\sqrt{ }$ \\
\hline Electromagnetic & $\sqrt{ }$ & $\sqrt{ }$ & $\sqrt{ }$ & $\sqrt{ }$ & $\sqrt{ }$ \\
\hline Strong & $\sqrt{ }$ & $\sqrt{ }$ & $\sqrt{ }$ & $\sqrt{ }$ & $\sqrt{ }$ \\
\hline Weak & $\times$ & $\times$ & $\times$ & $\times$ & $\sqrt{ }$ \\
\hline
\end{tabular}

From Table 1, it is interesting to ask that $C, P, T$ and $C P$ are broken, why not $C P T$ ? The immediate answer is that in the relativistic local quantum field theory, $C P T$ is invariance, so called the $C P T$ theorem 10. It holds based on three CPT conditions:

- Lorentz invariance,

- Hermiticity of the Hamiltonian,

- Locality.

It is clear that $C P T$ is a very fundamental symmetry. If it is found to be violated, there will be tremendous impact on our fundamental physics and at least one of the three conditions above must be given up. In string theory, $C P T$ can be spontaneously broken. In particular, a theoretical framework with Lorentz and CPT breaking terms has been formulated 11. The test of CPT invariance is thus of considerable theoretical and experimental interest.

In this talk, we will first briefly review $C P T$ tests based on the consequences of the CPT theorem and then study some possible $C P T$ tests due to exotic models in which one of the CPT conditions, hermiticity of the Hamiltonian, is lost.

\section{Some Possible CPT tests}

\subsection{Consequences of the CPT theorem}

With the CPT theorem in hand, it is possible to deduce many interesting consequences. For a system with the Lagrangian $\mathcal{L}$ and a local quantum field, we have

$$
\begin{aligned}
\Theta \mathcal{L}(\vec{x}, t) \Theta^{-1} & =\mathcal{L}^{\dagger}(-\vec{x},-t) \\
& =\mathcal{L}(-\vec{x},-t)
\end{aligned}
$$

for $\Theta \equiv C P T$. Note that Eq. (3) is guaranteed if $\mathcal{L}$ is Hermitian. From the table in Eq. (11), we find

$$
\Theta|p, \vec{p}, \vec{s}>=| \vec{p}, \vec{p},-\vec{s}>
$$


where $p(\bar{p})$ is particle (antiparticle) and $\vec{p}$ and $\vec{s}$ are the momentum and spin. In the following, we focus on five basic predictions due to the CPT theorem and we list them as tests by searching for their possible experimental deviations.

- Test 1: Mass equality between particle $(p)$ and antiparticle $(\bar{p})$, i.e., $m_{p}=m_{\bar{p}}$. It is obvious that the mass of a free particle should be that of the corresponding antiparticle due to the definition of charge conjugation. With interactions, it follows from CPT invariance even when $C$ breaks down. One can show this by considering a particle $p$ at rest with the z-component of angular momentum $m_{z}$ and Hamiltonian $H$ and one has

$$
T P C\left|p>_{m_{z}}=T P e^{i \alpha_{c}}\right| \bar{p}>_{m_{z}}=T e^{i \alpha_{c}}\left|\bar{p}>_{m_{z}}=e^{i \alpha_{c}}\right| \bar{p}>_{-m_{z}}
$$

where $e^{i \alpha_{c}}$ represents the phase factor under $C$. From Eq. (15) and $\Theta H \Theta^{-1}=H$, we get

$$
m_{p}=<p|H| p>_{m_{z}}=<p\left|\Theta^{-1} \Theta H \Theta^{-1} \Theta\right| p>_{m_{z}}=<\bar{p}|H| \bar{p}>_{-m_{z}}=m_{\bar{p}} .
$$

It is clear that if there exists a difference between $m_{p}$ and $m_{\bar{p}}$ for any $p$, CPT must be violated. Experimentally, stringent limits have been given in many systems. In particular, one has that 12

$$
\begin{gathered}
\left|m_{K^{0}}-m_{\bar{K}^{0}}\right| / m_{K}<10^{-18}, \\
\left|m_{e^{+}}-m_{e^{-}}\right| / m_{e}<8 \times 10^{-9} .
\end{gathered}
$$

The limit in Eq. (7) is the most accurate test for the CPT theorem.

- Test 2: Decay rate (lifetime) equality between $p$ and $\bar{p}$, i.e., $\Gamma_{p}=\Gamma_{\bar{p}}\left(\tau_{p}=\tau_{\bar{p}}\right)$. We follow the proof by Bigi and Sanda 13 , given by

$$
\begin{aligned}
\Gamma_{p} & =2 \pi \sum_{i} \delta\left(m_{p}-\varepsilon_{i}\right) \mid<i ; \text { out }\left|H_{\text {decay }}\right| p>\left.\right|^{2} \\
& =2 \pi \sum_{i} \delta\left(m_{p}-\varepsilon_{i}\right) \mid<i ; \text { out }\left|\Theta^{-1} \Theta H_{\text {decay }} \Theta^{-1} \Theta\right| p>\left.\right|^{2} \\
& =2 \pi \sum_{i} \delta\left(m_{p}-\varepsilon_{i}\right) \mid<\bar{i} ; \text { in }\left|H_{\text {decay }}\right| \bar{p}>\left.\right|^{2} \\
& =2 \pi \sum_{i} \delta\left(m_{p}-\varepsilon_{i}\right) \mid<\bar{i} ; \text { out }\left|H_{\text {decay }}\right| \bar{p}>\left.\right|^{2}=\Gamma_{\bar{p}},
\end{aligned}
$$

where the condition of complete sets of states has been used, i.e.,

$$
\sum_{i} \mid i ; \text { in }><i ; \text { in }\left|=\sum_{i}\right| i ; \text { out }><i ; \text { out } \mid=1 .
$$

Note that the decay rates in Eq. (9) are for the total decay rates and thus $\tau_{p}=\tau_{\bar{p}}$ since $\tau_{p}=1 / \Gamma_{p}$. Currently, the best search is also in the $K^{0}$ system with 12

$$
\left(\Gamma_{K^{0}}-\Gamma_{\bar{K}^{0}}\right) / m_{K_{\text {average }}}=(7.8 \pm 8.4) \times 10^{-18} .
$$


For the lifetime, one gets 12

$$
\left(\tau_{\mu^{+}}-\tau_{\mu^{-}}\right) / \tau_{\mu}=(2 \pm 8) \times 10^{-5} .
$$

On the other hand, if we neglect the final state interactions, we also expect the equality of partial decay rates between $p$ and $\bar{p}$. For examples, $\Gamma\left(\mu^{-} \rightarrow e^{-} \bar{\nu}_{e} \nu_{\mu}\right)=$ $\Gamma\left(\mu^{+} \rightarrow e^{+} \nu_{e} \bar{\nu}_{\mu}\right)$ and $\Gamma\left(K^{-} \rightarrow \pi^{-} \pi^{0}\right)=\Gamma\left(K^{+} \rightarrow \pi^{+} \pi^{0}\right)$.

- Test 3: Opposite sign of charge between $p$ and $\bar{p}$, i.e., $Q_{p}=-Q_{\bar{p}}$.

From the CPT theorem, one has that $\Theta \rho(x) \Theta^{-1}=-\rho(-x)$ with $\rho(x)$ being the charge density and one obtains

$$
\begin{aligned}
Q_{p} & =<p\left|\int d^{3} x \rho(x)\right| p>_{m_{z}}=<p\left|\Theta^{-1} \Theta \int d^{3} x \rho(x) \Theta^{-1} \Theta\right| p>_{m_{z}} \\
& =<\bar{p}\left|\int d^{3} x[-\rho(x)]\right| \bar{p}>_{-m_{z}}=-Q_{\bar{p}} .
\end{aligned}
$$

This prediction also holds to an extremely high accuracy 12. For example, $\mid Q_{e^{+}}+$ $Q_{e^{-}} \mid / e<4 \times 10^{-8}$.

- Test 4: Opposite sign of magnetic (electric) dipole moments between $p$ and $\bar{p}$,

$$
\text { i.e., } \mu_{p}=-\mu_{\bar{p}} \text { and } d_{p}=-d_{\bar{p}} \text {. }
$$

For a particle $p$ in uniform and static electromagnetic fields $\vec{E}$ and $\vec{B}$, we find

$$
\begin{aligned}
H & =-\mu_{p}<p|\vec{s} \cdot \vec{B}| p>-d_{p}<p|\vec{s} \cdot \vec{E}| p> \\
& =-\mu_{p}<p\left|\Theta^{-1} \Theta \vec{s} \cdot \vec{B} \Theta^{-1} \Theta\right| p>-d_{p}<p\left|\Theta^{-1} \Theta \vec{s} \cdot \vec{E} \Theta^{-1} \Theta\right| p> \\
& =-\mu_{p}<p\left|\Theta^{-1}(-\vec{s} \cdot \vec{B}) \Theta\right| p>-d_{p}<p\left|\Theta^{-1}(-\vec{s} \cdot \vec{E}) \Theta^{-1}\right| p> \\
& =\mu_{p}<\bar{p}|\vec{s} \cdot \vec{B}| \bar{p}>+d_{p}<\bar{p}|\vec{s} \cdot \vec{E}| \bar{p}>, \\
\Theta^{-1} H \Theta & =-\mu_{\bar{p}}<\bar{p}|\vec{s} \cdot \vec{B}| \bar{p}>-d_{\bar{p}}<\bar{p}|\vec{s} \cdot \vec{E}| \bar{p}>,
\end{aligned}
$$

which lead to $\mu_{p}=-\mu_{\bar{p}}$ and $d_{p}=-d_{\bar{p}}$ based on $\Theta^{-1} H \Theta=H$. It is interesting to note that from Eqs. (14) and (15), we conclude that both magnetic and electric dipole moments of Majorana neutrinos must vanish if CPT is conserved. Experimentally, by defining $\mu_{p} /\left(e \hbar / 2 m_{p}\right)-1=\left(g_{p}-1\right) / 2$, one has 12

$$
\left(g_{e^{+}}-g_{e^{-}}\right) / g_{\text {average }}=(-0.5 \pm 2.1) \times 10^{-12} .
$$

- Test 5: Opposite sign between transverse muon polarizations of $K^{+} \rightarrow \pi^{0} \mu^{+} \nu_{\mu}$ and $K^{-} \rightarrow \pi^{0} \mu^{-} \bar{\nu}_{\mu}$, i.e., $P_{T}\left(\mu^{+}\right)=-P_{T}\left(\mu^{-}\right)$.

The transverse muon polarization is associated with $\vec{s}_{\mu} \cdot\left(\vec{p}_{\pi} \times \vec{p}_{\mu}\right)$ which itself is a $P$-even and $T$-odd quantity. $P_{T}\left(\mu^{+}\right)$has been used to search for $C P$ violation induced by new physics 14 since it is vanishingly small in the standard model. The current experimental data is $P_{T}\left(\mu^{+}\right)=(-1.7 \pm 2.3 \pm 1.1) \times 10^{-3}$, given by the E246 collaboration at KEK 15 . 
The consequence of the CPT theorem in this test can be easily proved as follows:

$$
\begin{aligned}
& P_{T}\left(\mu^{+}\right)<\Theta^{-1} \Theta \vec{s}_{\mu^{+}} \cdot\left(\vec{p}_{\pi} \times \vec{p}_{\mu^{+}}\right) \Theta^{-1} \Theta>_{\mu^{+}} \\
= & P_{T}\left(\mu^{+}\right)<\Theta^{-1}\left[-\vec{s}_{\mu^{-}} \cdot\left(\vec{p}_{\pi} \times \vec{p}_{\mu^{-}}\right)\right] \Theta>_{\mu^{+}} \\
= & -P_{T}\left(\mu^{+}\right)<\vec{s}_{\mu^{-}} \cdot\left(\vec{p}_{\pi} \times \vec{p}_{\mu^{-}}\right)>_{\mu^{-}} \\
= & P_{T}\left(\mu^{-}\right)<\vec{s}_{\mu^{-}} \cdot\left(\vec{p}_{\pi} \times \vec{p}_{\mu^{-}}\right)>_{\mu^{-}} .
\end{aligned}
$$

Note that final state interactions give the same amount of contributions to $P_{T}\left(\mu^{-}\right)$ and $P_{T}\left(\mu^{-}\right)$but very small $O\left(10^{-6}\right)$.

\subsection{Models without hermiticity}

We now examine models without hermiticity as first suggested by Okun 16. In these models, Eq. (31) doesn't hold anymore and CPT could be broken. Moreover, the breaking down of hermiticity also breaks unitarity of $S$-matrix. We note that one cannot exclude this possibility from appearing in future microscopic quantum gravity as emphasized by Mavromatos 17. In this talk, we only concentrate on the CPT violating effects. In the following, we will present some examples as possible $C P T$ tests due to this exotic scenario.

- Test 1: Circular photon polarizations in $\Phi \rightarrow \gamma \gamma\left(\Phi=\pi^{0}, \eta\right) 16$

The most general effective interaction for $\Phi \rightarrow \gamma \gamma$ can be described by

$$
\begin{aligned}
\mathcal{L} & =g \Phi F_{\mu \nu} F_{\alpha \beta} \varepsilon^{\mu \nu \alpha \beta}+h \Phi F_{\mu \nu} F^{\mu \nu} \\
& =\mathcal{L}_{p}+\mathcal{L}_{s} .
\end{aligned}
$$

The term of $\mathcal{L}_{p}$ in Eq. (18) is the normal anomaly term which gives to the decay rates. In Table 2 we show the properties of $\mathrm{C}, \mathrm{P}$ and $\mathrm{T}$ for $\Phi, F_{\mu \nu} F_{\alpha \beta} \varepsilon^{\mu \nu \alpha \beta}$ and

Table 2. C, P and T transformations for $\Phi$, $F_{\mu \nu} F_{\alpha \beta} \varepsilon^{\mu \nu \alpha \beta}$ and $F_{\mu \nu} F^{\mu \nu}$

\begin{tabular}{|c|c|c|c|c|c|}
\hline Quantity & $P$ & $C$ & $T$ & $C P$ & $C P T$ \\
\hline \hline$\Phi$ & - & + & - & - & + \\
\hline$F_{\mu \nu} F_{\alpha \beta} \varepsilon^{\mu \nu \alpha \beta}$ & - & + & - & - & + \\
\hline$F_{\mu \nu} F^{\mu \nu}$ & + & + & + & + & + \\
\hline
\end{tabular}

$F_{\mu \nu} F^{\mu \nu}$. Note that

$$
F_{\mu \nu} F_{\alpha \beta} \varepsilon^{\mu \nu \alpha \beta} \propto \vec{E} \cdot \vec{B}, \quad F_{\mu \nu} F^{\mu \nu} \propto E^{2}-B^{2} .
$$

From Table 2 it is easy to see that the hermiticity of the Lagrangian in Eq. (18) requires that both $g$ and $h$ must be real numbers, i.e., $g=$ Reg and $h=$ Reh. In this case, CPT is a good symmetry for both $\mathcal{L}_{s}$ and $\mathcal{L}_{p}$. In general, Both $g$ and $h$ can be complex if hermiticity is broken. From the Lagrangian in Eq. (18), by summing 
over one of the photon polarization, we find that the squared amplitude is given by

$$
\begin{aligned}
|\mathcal{M}|^{2} \propto & \left(4|g|^{2}+|h|^{2}\right)\left[q \cdot k\left(q^{\mu} k^{\nu}+q^{\nu} k^{\mu}\right)-(q \cdot k)^{2} g^{\mu \nu}\right] \varepsilon_{\mu} \varepsilon_{\nu}^{*} \\
& +4 i \operatorname{Im}\left(g h^{*}\right)(q \cdot k) \varepsilon^{\mu \nu \alpha \beta} k_{\alpha} q_{\beta} \varepsilon_{\mu} \varepsilon_{\nu}^{*},
\end{aligned}
$$

where $q$ and $k$ are the 4-momenta of the two photon and $\varepsilon$ is one of the photon 4 -polarization vector. It is interesting to observe that in the rest frame of $\Phi$ the 2 nd term in Eq. (20) is related to

$$
\left(\vec{\varepsilon}_{1} \times \vec{\varepsilon}_{2}\right) \cdot \vec{k},
$$

which is the so called circular photon polarization. It is clear that with hermiticity no circular $\gamma$ polarization can be induced due to $\operatorname{Im}\left(g h^{*}\right)=0$. We now consider two extreme cases without hermiticity in Eq. (18), which are shown in Table 3 In

Table 3. $\mathrm{C}, \mathrm{P}$ and $\mathrm{T}$ transformations for $\mathcal{L}_{p}$ and $\mathcal{L}_{s}$ in the two cases.

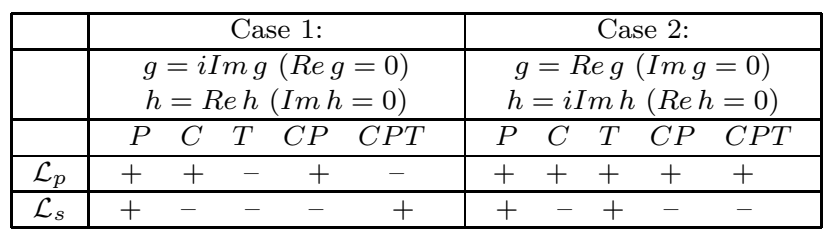

both cases, CPT is broken and a nonzero circular $\gamma$ polarzation is expected. It is interesting to note that imaginary parts of $g$ and $h$ can induce imaginary values of proton magnetic and electric dipole moments 16 , respectively, which of course are CPT violating quantities. Finally, we remark that the circular $\gamma$ polarzation can be also generated by the final state interaction such as the two-photon rescattering though electron box diagram [16. In this case, there is no CPT violation since $\operatorname{Im}\left(g h^{*}\right)=0$.

- Test 2: Circular photon polarization in ${ }^{1} S_{0}\left(e^{+} e^{-}\right) \rightarrow \gamma \gamma$

In Eqs. (14) and (15), we have assumed that $d_{p}$ is real. If CPT is broken, $d_{p}$ could be complex. If electron has an electric dipole moment, $d_{e}$, one can write the effective interaction as

$$
\mathcal{L}_{e d m}=-\frac{i}{2} d_{e} \bar{e} \sigma_{\mu \nu} \gamma_{5} e F^{\mu \nu},
$$

induced by loops. Note that hermiticity requires that $d_{e}$ must be real. Now, beside the tree QED diagram contribution, one gets contributions to $e^{+} e^{-} \rightarrow \gamma \gamma$ with replacing one vertex $\left(\gamma_{\mu}\right)$ in the tree diagram by the edm vertex in Eq. (22). The interferences between these two types of contributions lead to a non-zero value of circular $\gamma$ polarization 16 in Eq. (21) if $\operatorname{Im}\left(d_{e}\right) \neq 0$. Clearly, CPT must be broken.

- Test 3: Circular photon polarization in $\eta \rightarrow \pi^{+} \pi^{-} \gamma$

In Refs. 18] and 19, we have studied CP violation in $\eta \rightarrow \pi^{+} \pi^{-} \gamma$ with the CPT 
theorem. Here we shall discuss it without imposing the CPT symmetry. In the $\eta$ rest frame, The most general decay amplitude is given by

$$
\mathcal{M}=i m_{\eta}^{-2} E_{\gamma}\left[M \hat{k} \cdot\left(\vec{\varepsilon} \times \vec{p}_{+}\right)-E \vec{\varepsilon} \cdot \vec{p}_{+}\right],
$$

where $\vec{p}_{ \pm}$and $\vec{k}, E_{\gamma}$ and $\vec{\varepsilon}$ are momenta of $\pi^{ \pm}$and the phone momentum, energy and polarization, respectively. In the absence of final state interactions, $M$ and $E$ are purely real if the CPT theorem holds according to the table in Eq. (11). The squared amplitude from Eq. (23) is given by

$$
\begin{aligned}
|\mathcal{M}|^{2}= & m_{\eta}^{-4} E_{\gamma}^{2}\left\{|M|^{2}\left|\hat{k} \cdot\left(\vec{\epsilon} \times \vec{p}_{+}\right)\right|^{2}+|E|^{2}\left|\vec{\epsilon} \cdot \vec{p}_{+}\right|^{2}+\right. \\
& \left.E^{*} M\left[\hat{k} \cdot\left(\vec{p}_{+} \times \vec{\epsilon}\right)\right]\left(\vec{\epsilon} \cdot \vec{p}_{+}\right)^{*}+M^{*} E\left[\hat{k} \cdot\left(\vec{p}_{+} \times \vec{\epsilon}\right)\right]^{*}\left(\vec{\epsilon} \cdot \vec{p}_{+}\right)\right\} .
\end{aligned}
$$

It is easy to show 1819 that the circular photon polarization is found to be

$$
S_{2}\left(E_{\gamma}\right) \propto 2 \operatorname{Im}\left(E^{*} M\right) /\left(|E|^{2}+|M|^{2}\right),
$$

which is zero if both $E$ and $M$ are real. Phenomenologically, the decay rate of $\eta \rightarrow \pi^{+} \pi^{-} \gamma$ is described by a real $M$ term from the box-anomaly and resonance contributions 20]. Possible interactions which could yield the $E$ term in Eq. (23) were studied in Refs. 18, 19 In particular, a four-fermion operator was introduced, given by

$$
\mathcal{O}=\frac{1}{m_{\eta}^{3}} G \bar{s} i \sigma_{\mu \nu} \gamma_{5}(p-k)^{\nu} s \bar{u} \gamma^{\mu} u,
$$

where $u(s)$ stands for the up (strange) quark and $G$ is a dimensionless parameter originating from yet unknown short distance physics. The interaction in Eq. (26) leads to

$$
E \sim 2 e F\left(E_{\gamma}\right) G,
$$

where $F\left(E_{\gamma}\right)$ is a real form factor which is a function of $E_{\gamma}$. If $G$ contains an imaginary part, the circular $\gamma$ polarization $S_{2}$ in Eq. (25) is nonzero and CPT is broken. Note that it is possible that Eq. (26) can be generated by the electric dipole moment of the strange quark.

\section{Summary and Remarks}

There is no evidence of CPT Violation so far. The CPT violating effects such as the mass inequality between particle and antiparticle are found and expected to be vanishingly small due to the CPT theorem. There exist some exotic models which break hermiticity in the Lagrangians, in which CPT is also broken. Some consequences in these exotic models have been discussed. In particular, the circular photon polarizations have been studied. Finally, we remark that we have not examined CPT violating effects due to other CPT violating theories with hermiticity, in particular the recent ones based on the Lorentz and CPT violating terms in the modified Dirac equation 11 . 


\section{Acknowledgments}

This work was supported in part by the National Science Council of the Republic of China under Contract \#: NSC-93-2112-M-007-014.

\section{References}

1. H. Weyl, Symmetry (Princeton University Press; Reprint edition, 1983).

2. T. D. Lee and C. N. Yang, Phys. Rev. 104, 254 (1956).

3. E. Ambler et al., Phys. Rev. 105, 1413 (1957)

4. R. L. Garwin, L. M. Lederman and M. Weinrich, Phys. Rev. 105, 1415 (1957); J. J. Friedman and V. L. Telegdi, Phys. Rev. 105, 1681 (1957).

5. E. C. G. Sudarshan and R. E. Marshak, in Proc. Intern, Conf. on Mesons and Recently Discovered Particles (Padova, Venezia, 1957); R. P. Feynman and M. Gell-Mann, Phys. Rev. 109, 193 (1958).

6. J. H. Christenson et al., Phys. Rev. Lett. 13, 138 (1964).

7. Belle collaboration, Phys. Rev. Lett. 86, 2509 (2001); BaBar collaboration, Phys. Rev. Lett. 86, 2515 (2001).

8. CPLEAR Collaboration, Phys. Lett. B444, 43 (1998)

9. N. Cabibbo, Phys. Rev. Lett. 10, 531 (1963); M. Kobayashi and K. Maskawa, Prog. Theor. Phys. 49, 652 (1973).

10. O. W. Greenberg, arXiv:hep-ph/0309309 and references therein.

11. R. Bluhm, A. Kostelecky, C. Lane and N. Russell, arXiv:hep-ph/0306190 and references therein.

12. Particle Data Group, Phys. Lett. B592, 1 (2004).

13. I. I. Bigi and A. I. Sanda, CP Violation (Cambridge University Press, 2000).

14. G. Belanger and C. Q. Geng, Phys. Rev. D44, 2789 (1991).

15. M. Abe et al. [KEK-E246 Collaboration], Phys. Rev. Lett. 93, 131601 (2004)

16. L. B. Okun, arXiv:hep-ph/0210052

17. N. E. Mavromatos, arXiv:hep-ph/0504143 and references therein.

18. C. Q. Geng, J. N. Ng and T. H. Wu, Mod. Phys. Lett. A23, 1489 (2002).

19. C. Q. Geng and J. N. Ng, Phys. Scripta T99, 109 (2002).

20. See, e.g., J. Bijnens, G. Faldt and B. M. K. Nefkens, Phys. Scripta T99, 1-182 (2002); and references therein. A. Petrov, Phys. Atom. Nucl. 63, 499 (2000). 